\title{
Le programme de subventions de formation de chercheurs et d'action concertée (FCAC)*
}

\author{
LOUIS ROUSSEAU**
}

\section{AVANT-PROPOS}

Les lignes qui suivent présentent des souvenirs et des vues personnelles. La petite histoire, la présentation des statistiques véritables, l'étude des répercussions socio-économiques aussi bien que celle de l'impact psychologique et politique du programme restent à faire.

\section{Le contexte des années 1960 à 1970}

Où se situaient précisément la recherche et la formation des chercheurs au Québec avant et durant ces années? Sur combien et sur quels spécialistes notre société pouvait-elle compter pour l'essor qu'elle désirait prendre? Il n'était pas facile de répondre à ces questions et pourtant elles se situaient à la base même de décisions politiques et économiques.

Bien sûr, on se plaisait à souligner notre sous-développement par rapport à nos voisins du sud comme de l'ouest, et, à l'intérieur, par rapport à l'Université McGill dont la réputation faisait envie.

En réalité, beaucoup de chiffres manquaient alors et manquent encore en 1976. Mais il fallait tenter d'analyser la situation pour décider des actions à entreprendre.

Malgré l'existence de chercheurs isolés et de rares groupes de recherche dans les universités francophones du Québec, le Québec francophone manquait de structures favorables à l'épanouissement de la recherche. En effet, ses universités établies au sommet d'un système d'enseignement favorable à une certaine élite visaient à assurer avant tout l'alimentation des professions et bien peu le développement des connaissances par la recherche. Le manque de moyens y était aussi pour quelque chose.

Mais affirmer que le Québec francophone ne possédait pas de compétences, surtout sur la base de comparaisons avec le secteur anglophone, était une singulière déformation de la réalité. C'était un moyen peut-être de forcer la main aux divers gouvernements pour qu'ils se décident à injecter des capitaux du côté francophone, mais c'était aussi contribuer à entretenir un sentiment d'infériorité nationale; c'était permettre à certains employeurs d'affirmer qu'ils devaient recruter des spécialistes et des cadres parlant une autre langue puisque nous en avions si peu à offrir.

*Communication présentée à la conference annuelle de la Société canadienne pour l'étude de l'enseignement supérieur, Québec, juin, 1976.

**Directeur de la section Eau, Institut national de la recherche scientifique, Université du Québec. 
Pourtant un examen de la réalité démontrait que si nos universités francophones produisaient peu de doctorats ou de maîtrises, chaque année nous revenaient, s'ils ne trouvaient pas d'emploi outre frontière, de nombreux diplômés des grandes universités de Rome comme de Harvard, Munich, Paris ou Berkeley. Et les statistiques officielles des gouvernements et des universités n'en tenaient évidemment pas compte, et personne ne se souciait vraiment de mettre en valeur ces nouvelles ressources.

On constatait cependant l'importance de la production de doctorats à McGill, sans se soucier du fait que là aussi se formaient des francophones, à côté d'étrangers dont la majorité retournait dans leur pays!

Je crois qu'il reste à établir la statistique réelle de ces situations avec l'aide de démographes et d'historiens.

Mais pour corriger cette situation, le ministère de l'Education du Québec envisagea, avec le concours des universités francophones, d'octroyer en 1968 et en 1969 des subventions dites de "rattrapage", versées aux administrations universitaires. L'utilisation qui en fut faite n'a pas démontré que la recherche en ait entièrement bénéficié. D'autres mécanismes étaient cependant sur le point de voir le jour.

Au ministère de l'Education, où l'on s'employait à réfléchir sur l'expansion à donner à l'enseignement supérieur, l'effort portait aussi sur l'analyse des données disponibles, sur l'établissement de comparaison avec l'étranger, sur la rationalisation des investissements publics. La concertation et les contacts avec d'autres ministères, l'urgence de mettre en valeur toutes nos ressources québécoises pour accélérer le développement socio-économique d'une nation de plus en plus consciente de son existence allaient entraîner d'importantes décisions pour l'essor de la recherche universitaire.

\section{Certaines actions du gouvernement favorisant le développement de la recherche et de ses applications}

En 1968-69, les universités québécoises employaient environ 3,600 enseignants chercheurs, soit près de 1,200 chercheurs "équivalent temps plein", inscrivaient plus de 5,000 étudiants des deuxième et troisième cycles et les techniciens qui s'y rattachaient s'élevaient à quelque 2,500 personnes.

Cette même année, la part des crédits de recherche provenant de subventions indirectes du gouvernement québécois atteignait environ 29 millions de dollars dans un budget total des universités de 176 millions de dollars, soit $16.5 \%$ de celui-ci. Quant au financement direct de la recherche par le gouvernement fédéral, celui du Québec et les sources privées, il atteignait environ 25 millions de dollars. ${ }^{1}$

La part québécoise des subventions directes était cependant si faible en regard des autres sources que son impact politique et psychologique sur l'orientation des recherches était négligeable.

Une décision devait donc être prise de faire prendre conscience aux universités, ainsi qu'au gouvernement fédéral, de l'importance des subventions indirectes du Québec et aussi de mettre sur pied un programme de subventions directes supportant "des activités et une organisation de la recherche qui soient les mieux adaptées à la formation des chercheurs et des experts dont le Québec aura besoin, et . . en même temps qui viseraient à favoriser la réalisation de projets dont les résultats intéressent particulièrement le Québec."2 
D'autres décisons étaient aussi prises afin de limiter l'exode des étudiants: dorénavant les bourses du ministère de l'Education pour la préparation d'une maîtrise n'étaient accordées en principe que pour des études au Québec. Le financement des équipements universitaires et les subventions de fonctionnement étaient aussi considérablement accrus afin de développer un type de structures favorables à la recherche. Ces actions s'inscrivaient dans le même ensemble de décisions qui amenèrent la création de l'Institut de recherches de l'Hydro-Québec, du complexe scientifique hébergeant les laboratoires des ministères, du Centre de recherches industrielles du Québec, de la création de l'Institut national de la recherche scientifique, d'une Commission de la recherche au sein du Conseil des Universités, de la mise sur pied d'un Comité ministériel et d'un Conseil des politiques scientifiques. Il convient aussi de signaler le rôle que devaient jouer, dans ce contexte, les ententes entre la France et le Québec dans le développement de la recherche.

\section{Les caractéristiques du programme de subventions de formation de chercheurs et d'action concertée (FCAC)}

"Il est peut être nécessaire de lever dès maintenant une certaine ambiguïté en rappelant que dans l'esprit de tous ceux qui ont présidé à l'instauration du programme FCAC, ce dernier devait être un moyen par lequel le ministère de l'Education pourrait agir en vue de la coordination des efforts de recherche dans les universités québécoises et non pas seulement un moyen de rattrapage ou de soutien de certains projets spécifiques". 3

Le programme de subventions suit donc les éléments de politique concernant l'organisation générale de la recherche tel que défini par le gouvernement. La mise sur pied et le maintien d'équipes stables de recherche correspond au souci d'assurer l'expertise dans les domaines dont le Québec éprouve un besoin prioritaire. Il s'agit alors de supporter des thèmes et des programmes plutôt que des projets particuliers de recherche, assurant ainsi l'encadrement et la production de spécialistes de haut niveau. Dans ce cadre, les sciences de l'éducation bénéficient d'un traitement spécial. Ces objectifs complémentaires font par. tie de la stratégie élaborée pour la politique scientifique du Québec. ${ }^{4} \mathrm{Ce}$ souci du long terme n'exclut pas cependant la possibilité de supporter, non plus une équipe en vue du maintien de son expertise, mais un projet de recherche qui correspondrait à des préoccupations gouvernementales à plus court terme.

Au cours de l'exercice 1975-76, le gouvernement a décidé d'accorder des crédits importants à certains thèmes:

1. les ressources naturelles: l'océanologie, l'eau et l'énergie;

2. la technologie: dans le domaine des télécommunications, celui des lasers et celui des transports;

3. certains aspects de l'éducation;

\section{4. l'aménagement, le développement économique et la planification socio-économique et} territoriale.

De plus, il a appuyé des actions concertées entre les universités et le gouvernement, les organismes para-publics et l'entreprise privée, sur les lasers et les générateurs de plasmas.

Le programme de subventions FCAC accorde, en plus des crédits de fonctionnement, un support à l'équipement spécialisé. 
La notion d'équipe de recherche, chère au programme FCAC, repose sur l'existence d'au moins deux professeurs entourés d'autres chercheurs, d'assistants, de boursiers postdoctoraux, d'étudiants et de personnel de soutien. On estime qu'une équipe doit aussi avoir d'autres sources de revenus dont le programme FCAC tient alors compte pour estimer ses besoins. Au moins le tiers des étudiants faisant partie de l'équipe doit être supporté par une bourse d'étude.

Enfin, 70\% des équipes peuvent se voir accorder une présomption de renouvellement l'année suivante de la subvention afin d'assurer leur stabilité.

Si les équipes sont avant tout jugées par les pairs, leur productivité, - surtout pour celles qui sont bien établies - revêt une importance capitale. La valeur du projet soumis, les effets sur la société québécoise et la capacité à assurer des sujets de thèse de maîtrise et de doctorat comptent parmi les critères retenus par les jurys.

Aux subventions de recherche d'équipe s'ajoutent des subventions pour séminaires, congrès et colloques de recherche, ainsi que des subventions visant à assurer le support de centres de recherche dont le programme correspond à un des axes de développement susceptible de servir des intérêts communs à l'ensemble des universités du Québec. Ceci est particulièrement vrai pour les cas où une concentration des ressources est nécessaire et permet une programmation et un support logistique aux activités de recherche.

Le programme FCAC supporte aussi les organismes de service à la recherche: accélérateurs, stations expérimentales, laboratoires mobiles, banques de données, centres de documentation spécialisée, etc...

Enfin, des subventions dites générales sont aussi accordées aux universités pour tenir compte des imprévus de la recherche et amorcer des programmes nouveaux. Le montant de ces dernières subventions est proportionnel au total des subventions d'équipes reçues par chaque université.

\section{Commentaires sur le programme FCAC}

Si l'on peut affirmer que le programme FCAC s'est maintenant bien installé dans le milieu universitaire et qu'il y joue un rôle important non seulement en assurant le support des activités de recherche et de formation mais en permettant à tous ceux qui ont pu participer à ses jurys et à ses comités directeurs de se former aux mécanismes de critique et d'évaluation de projets, il reste que les crédits dont il a disposé ces dernières années sont devenus relativement insuffisants dans le contexte économique présent et qu'il semble que le gouvernement du Québec ne soit pas disposé à les augmenter dans l'immédiat.

On dit que les chercheurs n'ont pas su vendre leurs activités à la population et que ce déclin des crédits en est la conséquence. Il y a donc là matière à réflexion pour le programme FCAC.

D'un côté, je serais porté à affirmer que les politiques gouvernementales en matière de recherche sont encore trop mal définies et trop imprécises pour permettre aux chercheurs de s'en inspirer pour la définition de leurs objectifs de recherche.

D'autre part, les jurys m'apparaissent être en majorité dominés par des chercheurs fondamentalistes; le souci des besoins socio-économiques du Québec ne les anime pas assez. Peut-être doit-on susciter dans les milieux universitaires et gouvernementaux une réflexion sur cette question. L'action du gouvernement pourrait aussi s'étendre à la publicité qu'elle 
devrait accorder aux subventions et aux recherches qui touchent particulièrement les besoins de développement du Québec ainsi qu'aux résultats atteints par les chercheurs.

Finalement, il y aurait lieu de reconnaître officiellement en milieu universitaire qu'un rapport de recherche commanditée ou appliquée possède une valeur dans la carrière du chercheur aussi importante que la publication d'un article lu par quelques rares pairs! La valorisation de ce type de recherche ne m'apparaît pas avoir été un but suffisamment atteint par le programme FCAC. Il est, à mon avis, extrêmement regrettable que les jurys en tiennent peu ou pas du tout compte.

Si on me demandait de prédire un avenir au programme, je dirais qu'il en aura un à condition que les universités, les chercheurs et le gouvernement en fassent un outil véritable d'amélioration et de progrès de la société québécoise.

\section{Bibliographie}

1. "La recherche scientifique et technologique au Québec," Direction générale de l'enseignement supérieur, Ministère de l'Education. Document interne, 1969. 7pp.

2. Les subventions de formation de chercheurs et d'action concertée (FCAC). Brochure explicative. Commission de la recherche scientifique, Direction générale de l'enseignement supérieur, Ministère de l'Education, 1974. 28pp.

3. GAUVIN, L., Mémoire concernant la planification du programme FCAC. Commission de la recherche scientifique, Direction générale de l'enseignement supérieur Ministère de l'Education, 1973.

4. Les principes de la politique scientifique au Québec. Comité des politiques scientifiques du Québec. Gouvernement du Québec, 1974, 34pp. 